\title{
Clinical Features and Treatment Efficacy in CDKL5 Mutation-Related Epileptic Encephalopathy in the Infant
}

\author{
Chung Mo Koo, MD¹, Se Hee Kim, MD¹, Heung Dong Kim, MD¹, Joon Soo Lee, MD¹, Jong Rak Choi, MD², \\ Seung-Tae Lee, MD², Hoon-Chul Kang, MD \\ 'Department of Pediatrics, Severance Children's Hospital, Yonsei University College of Medicine, Seoul, Korea \\ ${ }^{2}$ Department of Laboratory Medicine, Severance Hospital, Yonsei University College of Medicine, Seoul, Korea
}

Received: February 20, 2019

Revised: May 7, 2019

Accepted: May 20, 2019

Corresponding author:

Hoon-Chul Kang, MD

Department of Pediatrics, Severance

Children's Hospital, Yonsei

University College of Medicine,

50-1 Yonsei-ro, Seodaemun-gu,

Seoul 03722, Korea

Tel: +82-2-2228-2050

Fax: +82-2-393-9118

E-mail: hipo0207@yuhs.ac
Purpose: Mutations in the cyclin-dependent kinase-like 5 (CDKL5) gene are associated with epileptic encephalopathy and severe cognitive impairment. We aim to characterize the association between this gene and treatment efficacy.

Methods: We retrospectively analyzed 10 patients who were treated at Severance

Children's Hospital for epileptic encephalopathy who were subsequently diagnosed with a CDKL5 mutation using next-generation sequencing.

Results: Electroencephalography (EEG) results showed generalized pattern abnormalities in 60\% (6/10) of patients with CDKL5 mutations. We analyzed the effects of three treatments, namely antiepileptic drugs (AEDs), ketogenic diet (KD), and steroids. A more than 50\% reduction in seizures was observed in $12 \%(1 / 8)$ of patients treated with clobazam. KD treatment proved ineffective in most cases. In addition, a more than 50\% reduction in seizures was observed in 57\% (4/7) of patients treated with steroids. EEG analysis of patients treated effectively with steroids revealed that 75\% (3/4) showed hypsarrhythmia and 25\% (1/4) showed focal epileptiform.

Conclusion: In this study, as in other studies, AEDs and KD did not effectively control seizures in most patients with a CDKL5 mutation. However, steroid therapy reduced the frequency of seizures in patients who also exhibited hypsarrhythmia. This suggests that steroid treatment is helpful in cases of hypsarrhythmia with CDKL5 mutations.

Keywords: Spasms, infantile; CDKL5 deficiency disorder; Epilepsy; Epileptic encephalopathy

\section{Introduction}

Mutations in the cyclin-dependent kinase-like 5 (CDKL5) gene are associated with severe cognitive impairments and early epileptic encephalopathy, such as infantile spasm. These mutations are mainly expressed in women $[1,2]$. Accordingly, the CDKL5 mutation was found in two female patients with severe cognitive impairment with an infantile spasm in 2003 [1]. The CDKL5 mutation has been reported in atypical Rett syndrome patients who report having seizures before 6 months of age [2,3]. However, unlike patients with typical Rett syndrome who had epileptic seizures before 3 years of age, those with CDKL5 mutations, on average, reported seizures 4 months earlier, and in many cases, lacked typical Rett syndrome features [3-5]. In 2013, Fehr et al. [6] reported that the CDKL5 mutation should be classified as an independent early epileptic encephalopathy, not atypical Rett syndrome. Seizures

CopyrightC 2019 Korean Child Neurology Society

This is an Open Access article distributed under the terms of the Creative Commons Attribution Non-Commercial License (http://creativecommons.org/licenses/by-nc/4.0/) which permits unrestricted non-commercial use, distribution, and reproduction in any medium, provided the original work is properly cited. 
with CDKL5 mutations in early infancy are typically characterized by tonic seizures or muscle contractions with vibration, followed by a clonic phase with a series of spasms that gradually turn into rhythmic distal myoclonic jerks [7]. There is, however, no characteristic electroencephalography (EEG) pattern in patients with a CDKL5 mutation [7-10]; EEG can present, initially, as normal background activity in patients with this mutation $[9,10]$. Although patients with CDKL5 mutations show a transient response to various antiepileptic drugs (AEDs), they are generally difficult to treat and are unresponsive to most intractable epilepsy treatment [11].

The purpose of this study was to analyze the characteristics of CDKL5 mutations in patients from a single center, as well as to confirm the efficacy of therapy.

\section{Materials and Methods}

We retrospectively analyzed 10 patients with CDKL5 pathogenic mutations, diagnosed at Severance Children's Hospital, Seoul, South Korea. Patients were reassessed at every outpatient clinic. The patient's response to the drug was categorized into four groups: (1) patient was seizure free for more than 6 months; (2) seizures were reduced by more than $50 \%$; (3) seizures were reduced by less than 50\%; or (4) no effect. If the patient does not affect the clinical symptoms, the drug or treatment method will be added or changed. In this case, we have assessed the treatment methods and drugs we want to investigate. In this study, when steroid was chosen as treatment options, we prescribed prednisolone. It was used at 40 to $60 \mathrm{mg} /$ day for 2 weeks and then tapered off for 2 weeks $[12,13]$.

All children underwent several analyses, including next-generation genetic sequencing, routine EEG recordings, and video EEG recordings. The EEG terminology used in this study is based on a paper published by Kane et al. [14] in 2017.

Genomic DNA extracted from all individual samples was used for library preparation and target capture using custom panels targeting candidate genes. The databases used for analysis and mutation analysis include online Mendel inheritance, Human Gene Mutation Database, ClinVar, dbSNP, 1000 Genome, Exome Aggregation Consortium, Exome Sequencing Project, and Korean Reference Genome Database. All pathogenic and possible pathogenic variants were identified by Sanger sequencing. All patients underwent video EEG recording at the first evaluation and routine EEG recording at the follow-up evaluation. Routine EEG recordings were performed for an average of 30 minutes and video EEG recordings were performed for more than 4 hours. Scalp electrodes were placed according to the International 10-20 system.
This study was approved by the Institutional Review Board of Severance Hospital (4-2016-0080). Informed consent was waived due to the retrospective nature of the study.

\section{Results}

We identified a CDKL5 pathogenic mutation in 10 patients (eight women, two men). Amongst all patients, the average age at seizure onset was 3.6 months (range, 0.6 to 11 ). Six patients with the mutation demonstrated generalized abnormalities in the first EEG recording; two patients showed hypsarrhythmic patterns, one showed EEG suppression patterns, two patients showed generalized sharp and wave discharges, and one patient showed generalized slow and disorganized background abnormalities. In total, four patients showed hypsarrhythmic patterns during the study period. Three patients demonstrated regional epileptiform discharges and one patient demonstrated regional paroxysmal fast activities on EEG. All patients exhibited motor seizures and six patients exhibited spasms. One of the patients who exhibited spasms did not show subsequent motor seizure. Brain magnetic resonance imaging data appeared normal in most cases, and we found no focal abnormalities. There was a significant delay in cognition in nine of the 10 patients who underwent cognitive testing. Details on genetic and clinical features of patients can be found in Tables 1 and 2 .

We compared the effects of AEDs such as valproic acid (VPA), clobazam (CLB), and vigabatrin (VGB) on seizures. Additionally, we evaluated the effects of steroids and ketogenic diets (KDs) on seizures. Eight of the 10 patients used VPA, six of whom reported no effects, and two reported a less than $50 \%$ reduction in the frequency of seizures. Nine patients used CLB, four of whom had a less than $50 \%$ seizure reduction, three had no effect, and one had a more than 50\% seizure reduction. Seven patients used VGB, five of whom reported no effects, and two reported a less than $50 \%$ re-

Table 1. Genetic features of 10 patients with CDKL5 mutation

\begin{tabular}{lcll}
\hline Pt & Inheritance & \multicolumn{1}{c}{ CDS/amino acid change } & \multicolumn{1}{c}{ Type of mutation } \\
\hline 1 & - & $\begin{array}{c}\text { c.2354dupA, } \\
\text { p.Lys786GlufsTer15 }\end{array}$ & Frameshift duplication \\
2 & De novo & c.511T>A, p.Tyr171Asn & Missense \\
3 & - & c.978-1G $>$ A & Splicing \\
4 & De novo & c.282+1G $<$ A & Splicing \\
5 & De novo & c. $175 \mathrm{C}>\mathrm{T}$, p.Arg59Ter & Nonsense \\
6 & De novo & c.513C $>$ A, p.Tyr171Ter & Nonsense \\
7 & - & c.458A $>$ T, p.Asp153Val & Missense \\
8 & De novo & c.145+2T $>$ A & Splicing \\
9 & - & c.403+1G $>$ A & Splicing \\
10 & De novo & c. $146-1 \mathrm{G}>\mathrm{T}$ & \\
\hline
\end{tabular}


duction in the frequency of seizures. Eight patients were on KDs, seven of whom reported no effect and only one of whom had a less than $50 \%$ reduction in the frequency of seizures (Table 3 ). In addition, seven patients used steroids, one of whom reported no seizures for more than 6 months, and three of whom reported a reduction in frequency of seizures by more than $50 \%$. EEG results in the patient who remained seizure-free for more than 6 months showed regional epileptiform when seizures first occurred (age, 0.6 months), but no slowing pattern. These three patients under steroids with a reduction of more than $50 \%$ in the frequency of seizures showed hypsarrhythmia on EEG.

\section{Discussion}

Patients with a CDKL5 mutation often present with epileptic encephalopathy, which is a challenging condition to treat, and has been reported to be only temporarily affected by AEDs $[11,15,16]$. Therefore, the treatment goal for these patients involves improving their quality of life and achieving, at minimum, a slight reduction in the frequency of seizures [11]. CDKL5 mutation is a causative mutation of infantile spasm, so treatment with steroid has also been tried in some cases $[12,13,15]$. One study reported that seizures were not completely abolished in patients with CDKL5 mutations, but $\mathrm{KD}$ and some drugs, such as VGB, have helped to reduce the frequency of seizures $[15,16]$.

Table 2. Clinical and EEG features of 10 patients with CDKL5 mutation

\begin{tabular}{|c|c|c|c|c|c|c|c|c|}
\hline Pt & Sex & $\begin{array}{l}\text { sz onset } \\
\text { age (mo) }\end{array}$ & ECD & sz type & 1st EEG finding & $\begin{array}{l}\text { Age at 1st } \\
\text { Hyps (mo) }\end{array}$ & Brain MRI & Cognitive function \\
\hline 1 & $\mathrm{~F}$ & 11 & WS, LGS & Spasm & Generalized sharp and wave discharges & 19 & $\mathrm{NL}$ & Significantly delayed \\
\hline 2 & $\mathrm{~F}$ & 3 & WS & Spasm & Normal & - & $\mathrm{NL}$ & Significantly delayed \\
\hline 3 & $\mathrm{~F}$ & 1.7 & EIEE & Myoclonic & Suppression burst & - & $\mathrm{NL}$ & Significantly delayed \\
\hline 4 & $\mathrm{~F}$ & 1 & WS, LGS & Spasm & Hyps & 3 & $\mathrm{NL}$ & Significantly delayed \\
\hline 5 & M & 5 & WS, LGS & Spasm & Hyps & 5 & $\mathrm{NL}$ & Significantly delayed \\
\hline \multirow[t]{2}{*}{6} & $\mathrm{~F}$ & 5 & WS & Spasm & $\begin{array}{l}\text { Generalized slow and disorganized } \\
\text { background activities }\end{array}$ & 9 & $\mathrm{NL}$ & Significantly delayed \\
\hline & & & & & Regional sharp wave discharges & & & \\
\hline 7 & $\mathrm{~F}$ & 3 & LGS & Spasm & Generalized sharp and slow wave & - & Atrophy & Significantly delayed \\
\hline 8 & M & 0.6 & EME & Myoclonic & $\begin{array}{l}\text { Regional sharp wave and regional } \\
\text { paroxysmal fast activities }\end{array}$ & - & $\mathrm{NL}$ & Significantly delayed \\
\hline 9 & $\mathrm{~F}$ & 4 & Focal epilepsy & Focal motor & Regional slowing & - & Atrophy & Significantly delayed \\
\hline 10 & $\mathrm{~F}$ & 2 & Focal epilepsy & GTC & Regional sharp wave discharges & - & $\mathrm{NL}$ & - \\
\hline
\end{tabular}

EEG, electroencephalography; CDKL5, cyclin-dependent kinase-like 5; Pt, patients; sz, seizure; ECD, electroclinical diagnosis; Hyps, hypsarrhythmia; MRI, magnetic resonance imaging; WS, West syndrome; LGS, Lennox-Gastaut syndrome; NL, normal; ElEE, early infantile epileptic encephalopathy; EME, early myoclonic encephalopathy; GTC, generalized tonic-clonic.

Table 3. Effect of treatment by reduction of seizure frequency for 10 patients with CDKL5 mutation

\begin{tabular}{|c|c|c|c|c|c|c|c|c|}
\hline $\mathrm{Pt}$ & Hyps & $\begin{array}{l}\text { Generalized } \\
\text { pattern EEG }\end{array}$ & Baseline sz freq & VPA & CLB & VGB & Steroid & KD \\
\hline 1 & + & + & Daily & Less than $50 \%$ & No effect & No effect & More than $50 \%$ & No effect \\
\hline 2 & - & - & 2 times/wk & No effect & Less than $50 \%$ & - & No effect & No effect \\
\hline 3 & - & + & Daily & Less than $50 \%$ & Less than $50 \%$ & Less than $50 \%$ & - & No effect \\
\hline 4 & + & + & Daily & No effect & Less than $50 \%$ & No effect & More than $50 \%$ & No effect \\
\hline 5 & + & + & Daily & No effect & - & Less than $50 \%$ & More than $50 \%$ & No effect \\
\hline 6 & + & + & Daily & No effect & Less than $50 \%$ & No effect & No effect & Less than $50 \%$ \\
\hline 7 & - & + & 3 times/wk & No effect & No effect & No effect & No effect & No effect \\
\hline 8 & - & - & Daily & - & - & No effect & sz free more than 6 mo & - \\
\hline 9 & - & - & Daily & No effect & More than $50 \%$ & - & - & No effect \\
\hline 10 & - & - & Daily & - & No effect & - & - & - \\
\hline
\end{tabular}

CDKL5, cyclin-dependent kinase-like 5; Pt, patients; Hyps, hypsarrhythmia; EEG, electroencephalography; sz, seizure; freq, frequency; VPA, valproic acid; CLB, clobazam; VGB, vigabatrin; KD, ketogenic diet. 
In the current study, we investigated 10 patients with the CDKL5 mutation, who were being treated with VPA $(n=8), \operatorname{CLB}(n=8)$, and/or VGB $(n=7)$. Five patients were being treated with a combination of all three drugs. Except for one patient who showed a more than $50 \%$ reduction in the frequency of seizures on CLB, most drugs yielded a less than 50\% reduction in the frequency of seizures. There was no effect in six of the eight patients on VPA, and five of the seven patients on VGB. With CLB, however, only three of the eight patients reported no effect, four patients reported a less than 50\% reduction, and one patient reported a less than $50 \%$ reduction. Based on these findings, CLB seems to be the most effective among the three drugs in reducing seizures.

We also studied the effect of a KD on seizure reduction. Eight patients were on a $\mathrm{KD}$, and seven of them did not show any reduction in seizures. This seems to be different from previous studies that ketone-producing diets are most helpful in reducing the frequency of seizures [15]. However, this study has been studied with a small number of patients, so further studies are needed.

Seven patients were treated with steroids for seizure control. One of them maintained a seizure-free condition for more than 6 months, and three patients reported a more than $50 \%$ reduction in seizures. EEG results showed that four patients had hypsarrhythmia, three of whom also demonstrated a more than 50\% reduction in seizures under steroid therapy. This suggests that steroid, a treatment option for infantile spasm, can also be a good treatment option for patients with CDKL5 mutations when they present with hypsarrhythmic EEG.

Contrary to previous studies, the AED and $\mathrm{KD}$ were not effective in CDKL5 mutation patients. However, in the case of hypsarrhythmic pattern on EEG, the steroid treatment showed a more than $50 \%$ seizure reduction effect (three-fourths). Currently, steroid therapy for infantile spasm is widely used and proven effective $[12,13]$. This study suggests that steroid treatment can also be helpful in cases of infantile spasm with a CDKL5 mutation who have a hypsarrhythmic EEG.

Due to the small number of patients included in this study and the limitations of the retrospective study, it is difficult to obtain reliable results, and some results show different results from previous studies. So, further studies will be needed in the future. In addition, this study evaluated the correlation with only interictal EEG pattern. This is also a limitation of this study and further research is needed.

\section{Conflicts of interest}

No potential conflicts of interest relevant to this article was reported.

\section{Acknowledgements}

This study was supported by a faculty research grant of Yonsei University College of Medicine (6-2015-0140).

This research was supported by a grant of the Korea Health Technology R\&D Project through the Korea Health Industry Development Institute (KHIDI), funded by the Ministry of Health and Welfare, Republic of Korea (grant number: HI18C0586).

\section{ORCID}

Hoon-Chul Kang, https:/ / orcid.org/0000-0002-3659-8847

\section{References}

1. Kalscheuer VM, Tao J, Donnelly A, Hollway G, Schwinger E, Kubart S, et al. Disruption of the serine/threonine kinase 9 gene causes severe $\mathrm{X}$-linked infantile spasms and mental retardation. Am J Hum Genet 2003;72:1401-11.

2. Evans JC, Archer HL, Colley JP, Ravn K, Nielsen JB, Kerr A, et al. Early onset seizures and Rett-like features associated with mutations in CDKL5. Eur J Hum Genet 2005;13:1113-20.

3. Mari F, Azimonti S, Bertani I, Bolognese F, Colombo E, Caselli $\mathrm{R}$, et al. CDKL5 belongs to the same molecular pathway of $\mathrm{MeCP} 2$ and it is responsible for the early-onset seizure variant of Rett syndrome. Hum Mol Genet 2005; 14:1935-46.

4. Scala E, Ariani F, Mari F, Caselli R, Pescucci C, Longo I, et al. CDKL5/STK9 is mutated in Rett syndrome variant with infantile spasms. J Med Genet 2005;42:103-7.

5. Bahi-Buisson N, Nectoux J, Rosas-Vargas H, Milh M, Boddaert N, Girard B, et al. Key clinical features to identify girls with CDKL5 mutations. Brain 2008;131(Pt 10):2647-61.

6. Fehr S, Wilson M, Downs J, Williams S, Murgia A, Sartori S, et al. The CDKL5 disorder is an independent clinical entity associated with early-onset encephalopathy. Eur J Hum Genet 2013;21:266-73.

7. Melani F, Mei D, Pisano T, Savasta S, Franzoni E, Ferrari AR, et al. CDKL5 gene-related epileptic encephalopathy: electroclinical findings in the first year of life. Dev Med Child Neurol 2011;53:354-60.

8. Buoni S, Zannolli R, Colamaria V, Macucci F, di Bartolo RM, Corbini L, et al. Myoclonic encephalopathy in the CDKL5 gene mutation. Clin Neurophysiol 2006;117:223-7.

9. Bahi-Buisson N, Kaminska A, Boddaert N, Rio M, Afenjar A, Gerard M, et al. The three stages of epilepsy in patients with CDKL5 mutations. Epilepsia 2008;49:1027-37.

10. Archer HL, Evans J, Edwards S, Colley J, Newbury-Ecob R, 
O'Callaghan F, et al. CDKL5 mutations cause infantile spasms, early onset seizures, and severe mental retardation in female patients. J Med Genet 2006;43:729-34.

11. Muller A, Helbig I, Jansen C, Bast T, Guerrini R, Jahn J, et al. Retrospective evaluation of low long-term efficacy of antiepileptic drugs and ketogenic diet in 39 patients with CDKL5-related epilepsy. Eur J Paediatr Neurol 2016;20:147-51.

12. Lux AL, Edwards SW, Hancock E, Johnson AL, Kennedy CR, Newton RW, et al. The United Kingdom Infantile Spasms Study (UKISS) comparing hormone treatment with vigabatrin on developmental and epilepsy outcomes to age 14 months: a multicentre randomised trial. Lancet Neurol 2005;4:712-7.

13. Lux AL, Edwards SW, Hancock E, Johnson AL, Kennedy CR, Newton RW, et al. The United Kingdom Infantile Spasms Study comparing vigabatrin with prednisolone or tetracosac- tide at 14 days: a multicentre, randomised controlled trial. Lancet 2004;364:1773-8.

14. Kane N, Acharya J, Benickzy S, Caboclo L, Finnigan S, Kaplan PW, et al. A revised glossary of terms most commonly used by clinical electroencephalographers and updated proposal for the report format of the EEG findings. Revision 2017. Clin Neurophysiol Pract 2017;2:170-85.

15. Moseley BD, Dhamija R, Wirrell EC, Nickels KC. Historic, clinical, and prognostic features of epileptic encephalopathies caused by CDKL5 mutations. Pediatr Neurol 2012;46:101-5.

16. Lim Z, Wong K, Olson HE, Bergin AM, Downs J, Leonard H. Use of the ketogenic diet to manage refractory epilepsy in CDKL5 disorder: experience of $>100$ patients. Epilepsia 2017;58:1415-22. 